\title{
Large D-instanton effects in string theory
}

\section{Boris Pioline $^{a}$ and Stefan Vandoren ${ }^{b}$}

${ }^{a}$ Laboratoire de Physique Théorique et Hautes Energies, CNRS UMR 7589, Université Pierre et Marie Curie, 4 place Jussieu, 75252 Paris Cedex 05, France

${ }^{b}$ Institute for Theoretical Physics and Spinoza Institute, Utrecht University, Leuvenlaan 4, 3508 TD Utrecht, The Netherlands

E-mail: pioline@lpthe.jussieu.fr, S.J.G.Vandoren@uu.nl

ABstract: By reduction along the time direction, black holes in 4 dimensions yield instantons in 3 dimensions. Each of these instantons contributes individually at order $\exp \left(-|Q| / g_{s}\right)$ to certain protected couplings in the three-dimensional effective action, but the number of distinct instantons is expected to be equal (or comparable) to the number of black hole micro-states, i.e. of order $\exp \left(Q^{2}\right)$. The same phenomenon also occurs for certain protected couplings in four dimensions, such as the hypermultiplet metric in type II string theories compactified on a Calabi-Yau threefold. In either case, the D-instanton series is therefore asymptotic, much like the perturbative expansion in any quantum field theory. By using a Borel-type resummation method, adapted to the Gaussian growth of the D-instanton series, we find that the total D-instanton sum has an inherent ambiguity of order $\exp \left(-1 / g_{s}^{2}\right)$. We further suggest that this ambiguity can be lifted by including Kaluza-Klein monopole or NS5-brane instantons.

KEYwords: Black Holes in String Theory, Nonperturbative Effects

ARXIV EPRINT: 0904.2303 
The large order behavior of perturbation theory is a telltale hint on the nature of nonperturbative effects in quantum mechanics and quantum field theory [1-3]. This also holds for string theory, and indeed, an estimate of the growth of string perturbation theory [4] led to the prediction of the existence of D-brane instantons [5] long before their actual construction [6-8]. D-instantons contribute to scattering amplitudes $\mathcal{A}$ in string theory on $\mathbb{R}^{1, d-1} \times Y$ schematically as

$$
\mathcal{A}_{\text {inst }}\left(g_{s}, t^{a}, \theta_{I}\right)=\sum_{Q^{I} \in L} \mu\left(Q^{I}, g_{s}, t^{a}\right) \exp \left(-\frac{1}{g_{s}} S_{\mathrm{Cl}}\left(Q, t^{a}\right)+2 \pi \mathrm{i} \theta_{I} Q^{I}\right),
$$

where $Q^{I}$ are the Ramond-Ramond charges in $d$ dimensions, valued in some rank $n$ lattice $L, \theta_{I}$ are the Ramond-Ramond axions, $t^{a}$ are the Neveu-Schwarz moduli, $S_{\mathrm{Cl}}\left(Q, t^{a}\right)$ is the classical action of the Euclideanized D-brane after extracting one power of the string coupling $g_{s}$, and $\mu$ is a function of $\left(Q, g_{s}, t^{a}\right)$ which behaves as a certain power of the string coupling constant as $g_{s} \rightarrow 0$ keeping the charges $Q^{I}$ and moduli $t^{a}$ fixed:

$$
\mu\left(Q, g_{s}, t^{a}\right)=g_{s}^{\alpha} \mu_{0}\left(Q, t^{a}\right)\left(1+\mathcal{O}\left(g_{s}\right)\right)
$$

Such instanton effects are typically negligible compared to perturbative corrections at small coupling $g_{s}$, but may become dominant for certain processes where perturbative contributions are forbidden due to non-renormalization theorems. In this note, we focus on "BPS saturated" couplings in the effective action of superstring theory, which receive perturbative corrections only up to a certain genus, and non-perturbative corrections from BPS instantons only, i.e. instantons (or multi-instantons) preserving a certain fraction of supersymmetry (see e.g. [9] for a review).

Our interest in this note is in the dependence of the "instanton measure" $\mu_{0}\left(Q, t^{a}\right)$ on the charges $Q^{I}$, and in the convergence properties of the D-instanton series (1). Since the classical action $S_{\mathrm{Cl}}\left(Q, t^{a}\right)$ typically scales linearly with $Q^{I}$, any faster-than-linear growth of $\log \mu$ as a function of the charges would imply that the series (1) would have zero radius of convergence, and should be treated as an asymptotic series.

In ordinary quantum field theory, $\mu_{0}\left(Q, t^{a}\right)$ can be calculated from the integration measure on the instanton moduli space and the one-loop fluctuation determinants around the instanton background. ${ }^{1}$ For BPS instantons in supersymmetric field theories, the bosonic and fermionic fluctuation determinants usually cancel, leaving only the integral of some characteristic class on the instanton moduli space.

In string theory, we do not know how to compute $\mu_{0}\left(Q, t^{a}\right)$ from first principles. In certain cases however, we may relate it to the indexed degeneracy of BPS solitons as follows [10]. Suppose that the compact manifold $Y$ is a product $X \times S^{1}$, and that the D-instanton in $\mathbb{R}^{d}$ is obtained by wrapping a Euclideanized D0-brane in $\mathbb{R}^{d+1}$ along the Euclidean time circle $S^{1}$ of radius $R$ (in particular, the D0-brane must have mass $M=S_{\mathrm{Cl}}\left(Q, t^{a}\right) /\left(2 \pi R g_{s}\right)$ and electric and magnetic charges $Q^{I}$, so as to reproduce the instanton action (1); the D0-brane may itself be obtained by wrapping $\mathrm{D} p$-branes on some

\footnotetext{
${ }^{1}$ The exponent $\alpha$ in (2) depends on the normalization of the external vertices, but usually not on $Q^{I}, t^{a}$; its precise value is irrelevant for our purposes.
} 
non-trivial $p$-cycle in $X)$. The instanton measure is given, up to a model-dependent normalization factor, ${ }^{2}$ by $^{3} \operatorname{Tr}\left[(-1)^{F} e^{-2 \pi R H}\right]$ in the D0-brane quantum mechanics with Hamiltonian $H$ [11]. Its large radius limit $R \rightarrow \infty$ defines the Witten index $\Omega(Q)$, i.e. the indexed degeneracy of the D0-brane bound state in $\mathbb{R}^{1, d}$. The latter is independent of both $g_{s}$ and $t^{a}$ by the attractor phenomenon (though it may jump across lines of marginal stability). Moreover, when the spectrum is discrete, the trace is independent of $R$, and therefore $\mu_{0}\left(Q, t^{a}\right)=\Omega(Q)$. If on the contrary the D-instanton in $\mathbb{R}^{d}$ originates from a D-instanton in $\mathbb{R}^{d} \times S^{1}$ smeared along $S^{1}$, T-duality along this circle maps it back to a Euclidean D0-brane wrapping $S^{1}$ of radius $l_{s}^{2} / R$, which reduces to a soliton in $\mathbb{R}^{1, d}$ in the limit $R \rightarrow 0$. In this case again, $\mu\left(Q, t^{a}\right)$ becomes equal to the indexed degeneracy of the T-dual D0-brane. Thus, in either of these two cases, we have [10]

$$
\mu_{0}\left(Q, t^{a}\right) \sim \Omega(Q)
$$

This relation may fail in cases where the D0-brane spectrum has a continuous part [12, 13]. This is for example the case of half BPS D-instantons in type IIB string theory in 9 dimensions, where the "bulk" contribution to the index precisely accounts for the discrepancy between the two sides of (3) [14]. More generally, this is the case when the charge vector $Q^{I}$ is non primitive. Similarly, we may expect that (3) breaks down at a wall of marginal stability; on either side of the wall however, we expect that (3) holds, as the same jump should affect the index and the instanton measure [15]. For our present purposes we shall only require that the two sides of (3) have the same asymptotic growth.

It should also be noted that when $d=3$, there are additional instantons in $\mathbb{R}^{3} \times S^{1} \times X$ which are of neither types above: Euclidean NS5-branes wrapped on $X$, and gravitational instantons asymptotic to $\mathbb{R}^{3} \times S^{1}$, also known as Kaluza-Klein monopoles or KK5-branes. The action of these instantons scales as $\tau_{2}^{2} V$ and $R^{2} \tau_{2}^{2} V$, where $V$ is the volume of $X$ in string units and $1 / \tau_{2} \propto g_{s}$ is the ten-dimensional string coupling. Their contributions are therefore exponentially suppressed compared to individual D-instanton contributions at weak coupling. We shall return to these effects momentarily.

Granting (3), it is now straightforward to estimate the prefactor $\mu_{0}\left(Q, t^{a}\right)$ at large charge $Q$ : under the standard assumption that the index $\Omega(Q)$ is equal or comparable to the exact degeneracy at strong gravitational coupling, we can use the black hole representation of the D-brane configuration to conclude that

$$
\mu_{0}\left(Q, t^{a}\right) \sim \exp \left[S_{\mathrm{BH}}(Q)\right]
$$

where $S_{\mathrm{BH}}(Q)$ is the Bekenstein-Hawking entropy. Our interest will be in situations where the gravitational solution is a single-centered 4D BPS black hole with a large horizon, tensored with a compact manifold $X$ (which may be a Calabi-Yau threefold, $K 3 \times T^{2}$ or $\left.T^{6}\right)$. For the horizon to be large in $4 \mathrm{D}$ Planck units, the $4 \mathrm{D}$ black hole must preserve

\footnotetext{
${ }^{2}$ We shall fix this proportionality factor in a specific example at the end of this note, when we discuss instantons in Calabi-Yau string compactifications.

${ }^{3}$ When the quantum mechanics has extended supersymmetry, one must include additional current insertions corresponding to the fermion bilinears appearing in the vertex $\mathcal{A}$.
} 
no more than 4 supercharges. The coupling $\mathcal{A}$ under study should therefore correspond to a two-derivative coupling in $3 \mathrm{D}$ vacua with 8 supercharges (e.g. the vector multiplet quaternionic metric in type II on $X \times S^{1}$ ), or a six-derivative coupling in a $3 \mathrm{D}$ vacua with 16 supercharges, or a fourteen-derivative coupling in 3D vacua with 32 supercharges. Even with this amount of supersymmetry, the existence of a single centered BPS solution typically requires some conditions on the total charge, e.g. $I_{4}(Q)>0$ in cases with 16 or 32 supercharges, where $I_{4}$ is the quartic polynomial such that $S_{\mathrm{BH}}(Q)=\pi \sqrt{I_{4}(Q)}$. In the opposite case $\left(I_{4}(Q)<0\right)$, there is usually no BPS black hole solution (although there may exist a non-BPS solution, not contributing to the index), and we set $S_{\mathrm{BH}}(Q)=0$. Combining (3) and (4), we conclude that

$$
\mu_{0}\left(Q, t^{a}\right)=a(Q) \exp \left[S_{\mathrm{BH}}(Q)\right]
$$

where $a(Q)$ grows at most like a power of $Q$ at large $Q$.

In any of the cases above, the Bekenstein-Hawking entropy of a single-centered BPS black hole solution is homogeneous of degree 2 in the electric and magnetic charges $Q^{I}$, and therefore the instanton sum (1) has zero radius of convergence. ${ }^{4}$ This does not mean that it is useless, but rather that it must be regarded as an asymptotic expansion. This is analogous to the usual situation in quantum field theory, where the perturbative expansion, of the form $^{5} \mathcal{A}(g)=\sum_{n \geq 0} n ! a_{n} g^{2 n}$ where $a_{n}$ is bounded by some power $n^{b}$ of the loop order, is assumed to be the asymptotic expansion of some non-perturbatively defined function describing the exact answer for the amplitude $\mathcal{A}$ (see e.g. [16] for a review). The truncated series $\mathcal{A}_{N}(g)=\sum_{0 \leq n \leq N-1} n ! a_{n} g^{2 n}$ should then approximate the exact result $\mathcal{A}(g)$ up to an error $\varepsilon$ which can be estimated to be of the order of the largest term in the sum, $\varepsilon=N ! N^{b}|g|^{2 N}$. This error is minimized upon choosing $N \sim 1 / g^{2}$ for $g$ small and $N$ large. At that optimum value, $\varepsilon \sim e^{-1 / g^{2}}$, the inherent ambiguity of the perturbative series.

Borel resummation consists in representing $n !=\frac{1}{g^{2}} \int_{0}^{\infty} \mathrm{d} t\left(t / g^{2}\right)^{n} e^{-t / g^{2}}$ and exchanging the $\int$ and $\sum$ signs. If the Borel transform $\mathcal{B}(t) \equiv \sum_{n>0} a_{n} t^{n}$ is well defined and regular everywhere on the positive real axis, the series $\mathcal{A}(g)$ is said to be "Borel summable", and its Laplace transform $\frac{1}{g^{2}} \int_{0}^{\infty} e^{-t / g^{2}} \mathcal{B}(t)$ produces a function $\tilde{\mathcal{A}}(g)$ with the same asymptotic expansion as $\mathcal{A}(g)$ in the sector $\operatorname{Re}\left(g^{2}\right)>0$. However, this procedure may be ambiguous due to singularities of $\mathcal{B}(t)$ at particular points or branch cuts in the Borel $t$ plane, typically along the real $t$-axis. To define the Laplace transform, one must choose a contour that avoids the singularities, but this choice of contour is not unique. Different contours lead to answers that differ by terms of order $\mathcal{O}\left(e^{-1 / g^{2}}\right)$, and a full non-perturbative definition of the quantum field theory is expected to fix these ambiguities, by relating them to computable instanton effects.

We can now apply the same line of reasoning to the divergent D-instanton series (1), where now the rôle of $g$ is played by $e^{-1 / g_{s}}$ and the growth of the Taylor coefficients is Gaussian rather than factorial. Our first task is to determine the optimal value of the

\footnotetext{
${ }^{4}$ This is in contrast to the usual Hagedorn divergence in perturbative string theory, which leads to a pole in the partition function.

${ }^{5}$ The case of perturbative string theory, corresponding to asymptotic series of the form $\mathcal{A}\left(g_{s}\right)=$ $\sum_{n}(2 n) ! a_{n} g_{s}^{n}[4,5]$, can be treated in the same way, upon replacing $g \rightarrow \sqrt{g_{s}}$.
} 
cut-off on $Q^{I}$ such that the error is minimized. Substituting (5) into (1) and dropping terms that scale like powers of $Q$, we find that this is achieved when

$$
\Sigma\left(Q, g_{s}, t^{a}\right) \equiv-S_{\mathrm{BH}}(Q)+\frac{1}{g_{s}} S_{\mathrm{Cl}}\left(Q, t^{a}\right)-2 \pi \mathrm{i} \theta_{I} Q^{I},
$$

is maximized as a function of $Q$. Since $S_{\mathrm{Cl}}\left(Q, t^{a}\right)$ scales linearly with $Q$, the optimum value of $Q$ is therefore of order $1 / g_{s}$ at small $g_{s}$, making the ambiguity of the asymptotic series of order $\exp \left(-\kappa / g_{s}^{2}\right)$.

To compute the coefficient $\kappa$, which will turn out to be positive, we need to specify the form of the instanton action $S_{\mathrm{Cl}}\left(Q, t^{a}\right)$. For definiteness, we restrict to the case of 3D backgrounds with 8 supercharges, e.g. type II string theory on $Y=X \times S^{1}$ where $X$ is a Calabi-Yau threefold. In conventions where $g_{s}$ is related to the 10 dimensional string coupling $1 / \tau_{2}$ via $1 / g_{s}^{2}=8 R^{2} V \tau_{2}^{2}$, the classical action of the $3 \mathrm{D}$ instanton, or the BPS mass of the $4 \mathrm{D}$ black hole, is proportional to the modulus of the central charge of the $\mathcal{N}=2$ Poincaré superalgebra,

$$
S_{\mathrm{Cl}}\left(Q^{I}, t_{a}\right)=2 \pi|Z(Q)|, \quad Z(Q) \equiv e^{K / 2} Q^{I} \mathcal{F}_{I},
$$

where $\mathcal{F}_{I}=\left(X^{\Lambda}, F_{\Lambda}\right)$ is the holomorphic symplectic section of $\mathcal{N}=2$ supergravity and $K=-\log \left(\mathrm{i} \mathcal{F}_{I} \overline{\mathcal{F}}^{I}\right)$ is the Kähler potential, related to the volume of $X$ in string units via $V=\frac{1}{8} e^{-K}$. Here and below, we use a notation in which indices are lowered using the symplectic form on $L \otimes \mathbb{C}$, e.g. i $\mathcal{F}_{I} \overline{\mathcal{F}}^{I}=\mathrm{i}\left(X^{\Lambda} \bar{F}_{\Lambda}-F_{\Lambda} \bar{X}^{\Lambda}\right)$.

Moreover, the Bekenstein-Hawking entropy can be computed by solving the "attractor equations" [17, 18] (see e.g. [19] for a review),

$$
\operatorname{Re}\left(\mathcal{F}^{I}\right)=Q^{I} \quad \Rightarrow \quad S_{\mathrm{BH}}(Q)=\frac{\mathrm{i} \pi}{4} \mathcal{F}_{I} \overline{\mathcal{F}}^{I} \geq 0
$$

To linearize the optimization problem over $Q^{I}$, we introduce a "twistor coordinate" $z$ [20] and replace (6) by

$$
\Sigma\left(Q, g_{s}, t^{a}, z\right) \equiv-S_{\mathrm{BH}}(Q)+\frac{\mathrm{i} \pi e^{K / 2}}{g_{s}}\left(\mathcal{F}_{I} z^{-1}-\overline{\mathcal{F}}_{I} z\right) Q^{I}-2 \pi \mathrm{i} \theta_{I} Q^{I},
$$

to be extremized over $Q^{I}$ and $z$. The extremal value of $z$ is proportional to the phase of the central charge,

$$
z=\mathrm{i} \sqrt{Z / \bar{Z}}
$$

Plugging this value back into (9), we recover (6). The extremization of (9) with respect to $Q^{I}$ amounts to a Legendre transform of the Bekenstein-Hawking entropy $S_{\mathrm{BH}}(Q)$. We define the Hesse potential to be the opposite of the Legendre transform of $S_{\mathrm{BH}}(Q)$ [21],

$$
\Sigma\left(\phi_{I}\right) \equiv\left\langle-S_{\mathrm{BH}}(Q)+\pi \phi_{I} Q^{I}\right\rangle_{Q^{I}},
$$

where $Q^{I}=\left(q_{\Lambda}, p^{\Lambda}\right)$ includes both the electric and magnetic charges, and $\phi_{I}=\left(\zeta^{\Lambda}, \tilde{\zeta}_{\Lambda}\right)$ includes both electric and magnetic potentials. Like the Bekenstein-Hawking entropy, the 
Hesse potential is homogeneous of degree two, and can be evaluated by using the "dual attractor equations" ${ }^{\prime}$ ([19], Ex. 8)

$$
\operatorname{Im}\left(\mathcal{F}_{I}\right)=-\phi_{I} \quad \Rightarrow \quad \Sigma(\phi)=\frac{\mathrm{i} \pi}{4} \mathcal{F}_{I} \overline{\mathcal{F}}^{I}
$$

Comparing (12) and (8) we conclude from that the Hesse potential is a positive function, equal to the Bekenstein-Hawking entropy function after replacing $Q^{I}$ with $\phi_{I}$. Applying (11) to (9), we obtain

$$
\left\langle\Sigma\left(Q, g_{s}, t^{a}, z\right)\right\rangle_{Q}=\frac{1}{g_{s}^{2}} \Sigma\left(\mathrm{i} e^{K / 2}\left(\mathcal{F}_{I} z^{-1}-\overline{\mathcal{F}}_{I} z\right)-2 \mathrm{i} g_{s} \theta_{I}\right),
$$

to be further extremized over $z$. Substituting (10) in (13) and expanding to leading order in $g_{s}$, we conclude that $\kappa$ is positive. Thus, the ambiguity of the D-instanton series is comparable to the expected contributions from KK5 or NS5-brane instantons wrapped on $X$.

Our second (related) task is to resum the D-instanton series in the region where $\operatorname{Re}\left(e^{K} / g_{s}^{2}\right)>0$ by generalizing the Borel-Laplace resummation method to the case of asymptotic series with Gaussian growth. For this purpose, we represent the exponential of the Bekenstein-Hawking entropy as a contour integral

$$
e^{S_{\mathrm{BH}}(Q)} \sim \int \mathrm{d} \phi_{I} e^{-\Sigma(\phi)+\pi \phi_{I} Q^{I}}
$$

where the variables $e^{\phi_{I}}$ can be thought of as the "Borel plane" variables. In writing (14) we remain imprecise about the specific choice of integration contour in the Borel plane, as it cannot be fixed without additional input. Here we require only that it selects the same saddle point as the Legendre transform (11), and neglect power corrections to the saddle point approximation.

The D-instanton sum (1) can now be rewritten as

$$
\begin{aligned}
\mathcal{A}\left(g_{s}, \theta\right) & =\sum_{Q \in L} \mu(Q) e^{-\frac{1}{g_{s}} S_{\mathrm{Cl}}\left(Q, t^{a}\right)+2 \pi \mathrm{i} \theta_{I} Q^{I}} \\
& =\int \mathrm{d} \phi_{I} e^{-\Sigma(\phi)}\left(\sum_{Q \in L} a(Q) e^{-\frac{1}{g_{s}} S_{\mathrm{Cl}}\left(Q, t^{a}\right)+2 \pi \mathrm{i}\left(\theta_{I}-\frac{\mathrm{i}}{2} \phi_{I}\right) Q^{I}}\right),
\end{aligned}
$$

where, in the second equality, we exchanged the summation over $Q$ with the integral over $\phi_{I}$, in effect implementing a "Borel-Gauss" resummation. According to our assumptions, the sum in bracket has now finite radius of convergence in $e^{\phi_{I}}$, but may have singularities away from the origin. Again, since $S_{\mathrm{Cl}}\left(Q, t^{a}\right)$ scales linearly in $Q$, we expect poles at $\phi_{I}^{*}+2 \mathrm{i} \theta_{I} \sim 1 / g_{s}$, leading to ambiguities of order $e^{-\Sigma\left(\phi^{*}\right)} \sim e^{-1 / g_{s}^{2}\left(1+\mathcal{O}\left(g_{s}\right)\right)}$ in the coupling $\mathcal{A}$.

\footnotetext{
${ }^{6}$ The terminology is only meant to emphasize the similarity of (12) and (8), and does not imply any physical attractor behavior for the potentials $\phi_{I}$.
} 
Just as in (9), in the case of 3D backgrounds with 8 supercharges it is convenient to write the exponential of the classical action as a contour integral over a "twistor coordinate" $z[20]$,

$$
\begin{aligned}
\mathcal{A}\left(g_{s}, \theta\right) \sim \int \mathrm{d} \phi_{I} e^{-\Sigma(\phi)} \int \frac{\mathrm{d} z}{z^{1+\delta}} \sum_{Q \in L} a(Q) \\
\quad \exp \left[-\left(\frac{\mathrm{i} \pi e^{K / 2}}{g_{s} z} \mathcal{F}_{I}-\frac{\mathrm{i} \pi e^{K / 2} z}{g_{s}} \overline{\mathcal{F}}_{I}-2 \pi \mathrm{i}\left(\theta_{I}-\frac{\mathrm{i}}{2} \phi_{I}\right)\right) Q^{I}\right],
\end{aligned}
$$

so that electromagnetic charges $Q^{I}$ now appear linearly in the exponent. The integral over $z$ is of Bessel type, with a saddle point at (10), and reproduces (15) up to irrelevant power corrections, irrespective of the value of $\delta$. We can now perform a Poisson resummation on $Q^{I}$

$$
\mathcal{A}\left(g_{s}, \theta\right) \sim \int \mathrm{d} \phi^{I} e^{-\Sigma(\phi)} \int \frac{\mathrm{d} z}{z^{1+\delta}}\left[\sum_{M_{I} \in L^{*}} b\left(\theta_{I}-\frac{\mathrm{i}}{2} \phi_{I}-\frac{e^{K / 2}}{2 g_{s} z} \mathcal{F}_{I}+\frac{e^{K / 2} z}{2 g_{s}} \overline{\mathcal{F}}_{I}-M_{I}\right)\right],
$$

where $b\left(M_{I}\right)$ is the Fourier transform of $a\left(Q^{I}\right)$; given our assumptions on $a(Q), b(M)$ is peaked around the origin $M_{I}=0$. For simplicity, we shall approximate $b(M)$ by a Dirac delta function, which would be exact if $a(Q)$ was equal to a constant. Thus, we obtain

$$
\mathcal{A}\left(g_{s}, \theta\right) \sim \int \frac{\mathrm{d} z}{z^{1+\delta}} \sum_{M_{I} \in L^{*}} e^{-\Sigma\left(\phi_{I}^{*}\right)}
$$

where

$$
\phi_{I}^{*}=-2 \mathrm{i}\left(\theta_{I}-M_{I}\right)+\frac{\mathrm{i} e^{K / 2}}{g_{s}}\left(\mathcal{F}_{I} z^{-1}-\overline{\mathcal{F}}_{I} z\right)
$$

and the sum only should include terms with $\operatorname{Re}\left[\Sigma\left(\phi^{*}\right)\right]>0$. To evaluate $\Sigma\left(\phi^{*}\right)$, we may now use (12). For example, setting $\theta_{I}=M_{I}$ and $z= \pm 1$, one finds

$$
\Sigma\left(\phi_{I}^{*}\right)=\frac{\mathrm{i} \pi}{4 g_{s}^{2}} e^{K} \mathcal{F}_{I} \overline{\mathcal{F}}^{I}=2 \pi R^{2} \tau_{2}^{2} V
$$

which is precisely the action of a Kaluza-Klein monopole wrapped on the Calabi-Yau threefold $X$. Unfortunately, we are not able to perform the remaining integral over $z$. Away from $\theta_{I}=M_{I}$, the quantum numbers $M_{I}$ give corrections of order $g_{s}$ to the KK5brane action, and should be interpretable as the charges of D-instantons bound to the KK5-brane. Of course, the classical action misses the minimal coupling to the NS-axion (or NUT potential) $\sigma$, which implies that the instanton responsible for the ambiguity in the Borel resummation should have zero total KK5-brane number, i.e. correspond to a supersymmetric bound state of a KK5-brane and an anti-KK5-brane. The fact that the Hesse potential (and therefore the Bekenstein-Hawking entropy function) controls the classical action of KK5-brane configurations is an interesting outcome of our analysis. It is perhaps not unexpected, since $\Sigma$ also controls the Kähler potential on the twistor space 
of the three-dimensional moduli space [20], while radially symmetric KK5-branes can be obtained as certain kind of geodesics on this space [22, 23].

In general, in addition to the power suppressed corrections to $S_{\mathrm{BH}}(Q)$, which are encoded in the Bekenstein-Hawking-Wald entropy, one expects further exponentially suppressed corrections. In the specific example of $\mathcal{N}=4$ string vacua in four dimensions, where the dyon degeneracies are known exactly [24], these corrections take the form

$$
\Omega(Q)=\sum_{k=1}^{\infty} \Omega_{k}(Q) e^{\frac{1}{k} S_{\mathrm{BH}}(Q)}
$$

where $\Omega_{k}(Q)$ is an infinite set of power corrections around each exponential term [25]. A similar form is also expected for $\mathcal{N}=2$ black holes, based on the Rademacher expansion [26, 27] of the elliptic genus of the MSW [28] superconformal field theory. The Borel-Gauss resummation discussed above can be applied to the terms with $k>1$ upon replacing $\Sigma \rightarrow k \Sigma$ in the previous derivation, and leads to exponentially suppressed corrections of order $e^{-k / g_{s}^{2}}$, characteristic of bound states of $k$ KK5-branes.

As a specific example of the general phenomenon discussed above, we now discuss the instanton corrections to the hypermultiplet moduli space in type II theories compactified on a Calabi-Yau three-fold $X$. As reviewed e.g. in [10], the hypermultiplet space in type IIB string theory receives instanton corrections from Euclidean D(-1), D1, D3 D5-branes wrapping complex cycles in $H^{\text {even }}(X, \mathbb{Z})$ (or more generally elements in the derived category of $X$, labelled by charges $Q^{I}$ in the K-theory lattice $L=K(X)$ ), and from NS5-branes wrapping $X$. The D-instanton corrections, to linear order around the one-loop corrected moduli space metric, are encoded in the "contact potential" [10, 29] (closely related to the hyperkähler potential on the Swann bundle over $\mathcal{M}[30,31]):^{7}$

$$
\begin{aligned}
e^{\Phi}= & \frac{1}{16 g_{s}^{2}}+\frac{\chi_{X}}{192 \pi} \\
& +\frac{1}{16 \pi^{2} g_{s}^{2}} \sum_{Q} n_{Q} \sum_{m>0} \frac{|Z(Q)|}{m} \cos \left(2 \pi m \theta_{I} Q^{I}\right) K_{1}\left(2 \pi m|Z(Q)| / g_{s}\right)+\ldots
\end{aligned}
$$

Comparing to $(1)$ and using $K_{1}(z) \sim(1+\mathcal{O}(1 / z)) e^{-z} \sqrt{\pi / 2 z}$, we read-off the D-instanton measure

$$
\mu\left(Q, g_{s}, t^{a}\right)=\left(1+\mathcal{O}\left(g_{s}\right)\right) \frac{|Z(Q)|^{1 / 2}}{64 \pi^{2} g_{s}^{3 / 2}} \sum_{m \mid Q^{I}} m^{-2} n_{Q / m} .
$$

When $Q^{I}$ is a primitive vector in the lattice $L$, only $m=1$ contributes to the sum, and therefore $\mu \sim n_{Q}$ up to normalization factors. It is worthwhile to note that the same sum over divisors appears for $\mathrm{D}(-1)$-instantons in 10 dimensions [11]. The NS5-brane contributions are not well understood at present, although some suggestions have been made [32-34].

The D-instanton measure $\mu$ may be related to indexed degeneracies of four-dimensional BPS black holes as explained above (3). Specifically, the hypermultiplet moduli

\footnotetext{
${ }^{7}$ To translate into the notations of [10], recall that in this $4 \mathrm{D}$ set-up, $1 / g_{s}^{2}=8 \mathrm{~V} \tau_{2}^{2}=e^{-K} \tau_{2}^{2}$.
} 
space is unaffected by reduction to 3 dimensions on a circle $S_{1}$ of radius $\tilde{R}$. Under Tduality along that circle, it is identified with the vector multiplet moduli space in type IIA string theory compactified on the same Calabi-Yau three-fold $X$ times the T-dual $S_{1}$ of radius $R=1 / \tilde{R}$. The afore mentioned D-instantons are T-dual to BPS black holes in 4 dimensions, obtained by wrapping D0,D2,D4,D6 branes on complex cycles in the homology class $Q \in L$ times the circle $S_{1}(R)$. Thus, the D-instanton measure $\mu_{0}$ (after dropping the moduli dependent prefactor in (23)) should be equal to the indexed degeneracy of a four-dimensional black hole in the same homology class, or in mathematical terms, to the generalized Donaldson-Thomas invariant [35, 36] $n_{Q}$ [31] (for $Q \in H^{0}+H^{2}$, they must reduce to the genus 0 Gopakumar-Vafa invariants of $X[37])$.

The divergence of the resulting D-instanton series (22) has often been raised as an objection against the equality of the instanton measure and the indexed degeneracy of BPS black holes, and therefore against the usefulness of the hypermultiplet moduli space as a book-keeping device for microscopic degeneracies of BPS black holes [22]. As we have argued in this note, this objection is not as fatal as it once seemed: in spite of its Gaussian growth, it is perfectly consistent to treat the D-instanton series as an asymptotic series, with an inherent ambiguity of order $e^{-1 / g_{s}^{2}}$. This ambiguity is precisely of the correct magnitude to be cancelled by KK5-brane contributions to the vector multiplet branch, or by NS5-brane contributions to the hypermultiplet branch. Realizing this scenario will require a far-reaching extension of the framework of $[10,15,35,36]$ into the NS5/KK5 sector. In particular, one may wonder whether NS5/KK5-brane contributions are themselves Borel summable, or whether yet more exotic effects are still looming behind.

\section{Acknowledgments}

It is a pleasure to thank S. Alexandrov, M. Berkooz, J. de Boer, G. 't Hooft, G. Moore, A. Neitzke, F. Saueressig and A. Strominger for discussions. B.P. is grateful to the Spinoza institute for hospitality during the completion of this work. The research of B.P. is supported in part by ANR (CNRS-USAR) contract no.05-BLAN-0079-01.

\section{References}

[1] F.J. Dyson, Divergence of perturbation theory in quantum electrodynamics, Phys. Rev. 85 (1952) 631 [SPIRES].

[2] E. Brézin, J.C. Le Guillou and J. Zinn-Justin, Perturbation theory at large order. 1. The $\phi^{2 N}$ interaction, Phys. Rev. D 15 (1977) 1544 [SPIRES].

[3] E. Brézin, J.C. Le Guillou and J. Zinn-Justin, Perturbation theory at large order. 2. Role of the vacuum instability, Phys. Rev. D 15 (1977) 1558 [SPIRES].

[4] D.J. Gross and V. Periwal, String perturbation theory diverges, Phys. Rev. Lett. 60 (1988) 2105 [SPIRES].

[5] S.H. Shenker, The strength of nonperturbative effects in string theory, talk presented at the Cargese Workshop on Random Surfaces, Quantum Gravity and Strings, May 28-June 1, Cargese, France (1990). 
[6] M.B. Green and J. Polchinski, Summing over world sheet boundaries, Phys. Lett. B 335 (1994) 377 [hep-th/9406012] [SPIRES].

[7] J. Polchinski, Combinatorics of boundaries in string theory, Phys. Rev. D 50 (1994) 6041 [hep-th/9407031] [SPIRES].

[8] J. Polchinski, Dirichlet-branes and Ramond-Ramond charges, Phys. Rev. Lett. 75 (1995) 4724 [hep-th/9510017] [SPIRES].

[9] E. Kiritsis, Duality and instantons in string theory, hep-th/9906018 [SPIRES].

[10] S. Alexandrov, B. Pioline, F. Saueressig and S. Vandoren, D-instantons and twistors, JHEP 03 (2009) 044 [arXiv:0812.4219] [SPIRES].

[11] M.B. Green and M. Gutperle, Effects of D-instantons, Nucl. Phys. B 498 (1997) 195 [hep-th/9701093] [SPIRES].

[12] S. Sethi and M. Stern, D-brane bound states redux, Commun. Math. Phys. 194 (1998) 675 [hep-th/9705046] [SPIRES].

[13] P. Yi, Witten index and threshold bound states of D-branes, Nucl. Phys. B 505 (1997) 307 [hep-th/9704098] [SPIRES].

[14] M.B. Green and M. Gutperle, D-instanton partition functions, Phys. Rev. D 58 (1998) 046007 [hep-th/9804123] [SPIRES].

[15] D. Gaiotto, G.W. Moore and A. Neitzke, Four-dimensional wall-crossing via three-dimensional field theory, arXiv:0807.4723 [SPIRES].

[16] M. Beneke, Renormalons, Phys. Rept. 317 (1999) 1 [hep-ph/9807443] [SPIRES].

[17] S. Ferrara, R. Kallosh and A. Strominger, $N=2$ extremal black holes, Phys. Rev. D 52 (1995) 5412 [hep-th/9508072] [SPIRES].

[18] A. Strominger, Macroscopic entropy of $N=2$ extremal black holes, Phys. Lett. B 383 (1996) 39 [hep-th/9602111] [SPIRES].

[19] B. Pioline, Lectures on on black holes, topological strings and quantum attractors, Class. Quant. Grav. 23 (2006) S981 [hep-th/0607227] [SPIRES].

[20] A. Neitzke, B. Pioline and S. Vandoren, Twistors and black holes, JHEP 04 (2007) 038 [hep-th/0701214] [SPIRES].

[21] G. Lopes Cardoso, B. de Wit, J. Kappeli and T. Mohaupt, Black hole partition functions and duality, JHEP 03 (2006) 074 [hep-th/0601108] [SPIRES].

[22] M. Günaydin, A. Neitzke, B. Pioline and A. Waldron, BPS black holes, quantum attractor flows and automorphic forms, Phys. Rev. D 73 (2006) 084019 [hep-th/0512296] [SPIRES].

[23] M. Günaydin, A. Neitzke, B. Pioline and A. Waldron, Quantum attractor flows, JHEP 09 (2007) 056 [arXiv:0707.0267] [SPIRES].

[24] R. Dijkgraaf, E.P. Verlinde and H.L. Verlinde, Counting dyons in $N=4$ string theory, Nucl. Phys. B 484 (1997) 543 [hep-th/9607026] [SPIRES].

[25] N. Banerjee, D.P. Jatkar and A. Sen, Asymptotic expansion of the $N=4$ dyon degeneracy, JHEP 05 (2009) 121 [arXiv:0810.3472] [SPIRES].

[26] R. Dijkgraaf, J.M. Maldacena, G.W. Moore and E.P. Verlinde, A black hole Farey tail, hep-th/0005003 [SPIRES]. 
[27] J. de Boer, M.C.N. Cheng, R. Dijkgraaf, J. Manschot and E. Verlinde, A Farey tail for attractor black holes, JHEP 11 (2006) 024 [hep-th/0608059] [SPIRES].

[28] J.M. Maldacena, A. Strominger and E. Witten, Black hole entropy in M-theory, JHEP 12 (1997) 002 [hep-th/9711053] [SPIRES].

[29] S. Alexandrov, D-instantons and twistors: some exact results, arXiv:0902.2761 [SPIRES].

[30] B. de Wit, M. Roček and S. Vandoren, Hypermultiplets, hyperkähler cones and quaternion-Kähler geometry, JHEP 02 (2001) 039 [hep-th/0101161] [SPIRES].

[31] S. Alexandrov, B. Pioline, F. Saueressig and S. Vandoren, Linear perturbations of quaternionic metrics. II. The quaternionic-Kähler case, arXiv:0810.1675 [SPIRES].

[32] R. Dijkgraaf, E.P. Verlinde and M. Vonk, On the partition sum of the NS five-brane, hep-th/0205281 [SPIRES].

[33] A. Kapustin, Gauge theory, topological strings and S-duality, JHEP 09 (2004) 034 [hep-th/0404041] [SPIRES].

[34] B. Pioline and D. Persson, The automorphic NS5-brane, arXiv:0902.3274 [SPIRES].

[35] D. Joyce, Holomorphic generating functions for invariants counting coherent sheaves on Calabi-Yau 3-folds, Geom. Topol. 11 (2007) 667.

[36] M. Kontsevich and Y. Soibelman, Stability structures, motivic Donaldson-Thomas invariants and cluster transformations, arXiv:0811.2435.

[37] D. Robles-Llana, M. Roček, F. Saueressig, U. Theis and S. Vandoren, Nonperturbative corrections to $4 D$ string theory effective actions from $\mathrm{SL}(2, Z)$ duality and supersymmetry, Phys. Rev. Lett. 98 (2007) 211602 [hep-th/0612027] [SPIRES]. 